\title{
Bioavailability assessment of topical delivery systems: effect of inter-subject variability on relative in vitro deliveries of minoxidil and hydrocortisone from solution and ointment formulations
}

\author{
Chia-Ming Chiang ${ }^{1}$, G.L. Flynn ${ }^{1}$, N.D. Weiner ${ }^{1}$ and G.J. Szpunar ${ }^{2}$ \\ ${ }^{\prime}$ College of Pharmacy, University of Michigan, Ann Arbor, MI 48109 (U.S.A.) and ${ }^{2}$ The Upjohn Company, Kalamazoo, MI (U.S.A.)
}

(Received 11 April 1988)

(Modified version received 27 June 1988)

(Accepted 13 July 1988)

Key words: Topical; Vehicle; Thin application; Diffusion; Permeability; Flux

Summary

The present study demonstrates that in vitro comparisons of delivery vehicles are reliable as long as one performs the studies on skin samples taken from the same cadaver section of skin. The in vitro rates of delivery of minoxidil and hydrocortisone from an ointment and a propylene glycol/ethanol/water vehicle through skin sections taken from the same and different cadaver abdomens are specifically compared. When tested on skins from 8 human cadavers, the fluxes of minoxidil suspended in the ointment ranged from $0.37 \times 10^{-5} \mathrm{mg} / \mathrm{cm}^{2} / \mathrm{h}$ to $9.2 \times 10^{-5} \mathrm{mg} / \mathrm{cm}^{2} / \mathrm{h}$, a 25 -fold spread, and the fluxes of minoxidil from the propylene glycol/ethanol/water vehicle ranged from $1.25 \times 10^{-5} \mathrm{mg} / \mathrm{cm}^{2} / \mathrm{h}$ to $18.7 \times 10^{-5} \mathrm{mg} / \mathrm{cm}^{2} / \mathrm{h}$, a 15 -fold spread. However, when the ratios of fluxes between these two formulations were calculated for individual subjects, the values ranged from 1.3 to 3.5 (only a 3-fold spread). Four human cadaver sections were used to evaluate the delivery of hydrocortisone. The flux values ranged from 696 to $1335 \mathrm{cpm} / \mathrm{cm}^{2} / \mathrm{h}$ for the ointment and 946 to $1291 \mathrm{cpm} / \mathrm{cm}^{2} / \mathrm{h}$ for the propylene glycol/ethanol/water vehicle (up to a $2-f$ old spread). When the ratios of fluxes between these two formulations were calculated for each individual subject, the values ranged from 0.96 to 1.39 . The results with these two compounds suggest that even in the unavoidable presence of large inter-subject variations in the cadaver skin, the influence of vehicle on drug delivery performance can be determined. In addition to these findings concerning intra-subject variability, it was observed that the flux of minoxidil from the ointment attains a steady-state after an initial lag period of between 6 and $8 \mathrm{~h}$ but steady delivery is not apparent for the minoxidil solution, suggesting that for the solution formulation the thermodynamic activity of the drug changes markedly as the experiment proceeds. No comparable curvature is seen for the hydrocortisone solution presumably because its thermodynamic activity is little changed during the vehicle's evaporation.

\section{Introduction}

As previously shown (Chiang et al., 1988), under rigorously defined conditions the delivery per-

Correspondence: G.L. Flynn, College of Pharmacy, University of Michigan, Ann Arbor, MI 48109, U.S.A. formance of a series of topical formulations can be compared by an in vitro technique. Of importance, formulation comparisons were based on data obtained from human cadaver membranes prepared from a single section of skin because a number of previous studies had shown that the flux values obtained with different skins vary considerably (Shaw et al., 1980; Southwell et al., 
1984). Since a given sample of cadaver skin is limited in size, it is obviously impossible to compare a series of formulations using a single section of skin. Moreover, it remains uncertain whether one can generalize results obtained within a piece of skin.

The purpose of this study is to determine if, in spite of large inter-subject variations in flux rates of a given drug in a given formulation, reliable in vitro appraisals of the influences of formulation on drug delivery are possible, provided one performs the studies on closely paired skin samples taken from the same cadaver section. An attempt is also made to see if parallel trends in the permeation of a drug from a series of formulations exist across skins from different subjects. In order to fairly assess these two points, the fluxes of minoxidil and hydrocortisone from two formulations, a propylene glycol/ethanol/water solution and an ointment, have been evaluated using different human cadaver skin samples.

\section{Materials and Methods}

\section{Materials}

Minoxidil, USP milled, was supplied by the Upjohn Co. (Kalamazoo, MI). The [pyrimidine-2${ }^{14} \mathrm{C}$ ]minoxidil was obtained from the same source with a radiochemical purity of $99 \%$ and a specific activity of $15.79 \mathrm{mCi} / \mathrm{mmol}$. [1,2- ${ }^{3} \mathrm{H}[\mathrm{Hydrocorti-}$ sone (Amersham, Arlington Height, IL) had a radiochemical purity of $97 \%$ and a specific activity of $45 \mathrm{Ci} / \mathrm{mmol}$. The mineral oil (Mallinkrodt, Paris, KY), white petrolatum (CMC, Smyrna, TN) and white wax required to prepare the ointment were used as received. All other chemicals used were reagent grade.

\section{Preparation of minoxidil and hydrocortisone formu- lations}

A recrystallized mixture of radiolabelled and cold minoxidil was prepared by the following procedure. About $10 \mathrm{mg}$ of $\left[{ }^{14} \mathrm{C}\right]$ minoxidil was mixed together with unlabeled minoxidil (about $200 \mathrm{mg}$ ) in a $200 \mathrm{ml}$ flask and $6 \mathrm{ml}$ warm ethanol solution were added to dissolve the mixture. Hexane (about $160 \mathrm{ml}$ ) was then added to initiate the recrystallization of the minoxidil. The flask was chilled in an ice bath for $4 \mathrm{~h}$ and the recrystallized minoxidil powder was collected under vacuum filtration and placed in a desiccator overnight to facilitate removal of the solvent. The resultant recrystallized minoxidil had a specific activity of about $3 \mu \mathrm{Ci}$ / mg.

The ointment base was prepared from petrolatum/mineral oil/white wax $(50: 45: 5, \mathrm{w} / \mathrm{w})$ by heating these components at $65^{\circ} \mathrm{C}$ and stirring the melt until the preparation cooled and congealed. The solution vehicle was prepared by mixing propylene glycol/ethanol/water (20:63.2: $16.8, \mathrm{v} / \mathrm{v})$. Since the amount of radiolabelled minoxidil available was limited, the $2 \%$ minoxidil ointment was prepared by incorporating the recrystallized minoxidil into a suitable amount of the ointment contained on a watch glass with the aid of a small pestle. The $2 \%$ minoxidil solution was prepared by incorporating recrystallized minoxidil into a suitable volume of the vehicle.

In a number of the experiments, small amounts of labeled hydrocortisone (approximately $3 \mu \mathrm{Ci}$ ) were incorporated into the two different minoxidil formulations. The quantities of this drug were kept small to ensure that its solubility was not exceeded in either formulation.

\section{Permeation studies}

Abdominal skin obtained from 8 Caucasian human cadavers with an average age of 62 years was used. The abdominal site was chosen because it is expansive and because it has been reported to be less variable in permeability than other body sites (Southwell et al., 1984). No attempt was made to determine whether age influenced the permeation of drugs from the formulations tested. The skin samples (about $200 \mu \mathrm{m}$ in thickness) were harvested as thin sheets with the aid of a dermatome. They were stored at $-10^{\circ} \mathrm{C}$ in a freezer prior to use. The frozen skins were thawed before use and cut into $4 \mathrm{~cm}^{2}$ sections. Depending upon the size of the skin sample acquired, approximately 5-10 membranes could be prepared for mounting into diffusion cells.

For determining the permeation of drugs from the ointment, the thin film application technique previously described by Chiang et al. (1988) was used. Briefly, a section of skin was placed on a 
larger piece of Parafilm with the stratum corneum facing upwards. A template $(200 \mu \mathrm{m}$ thick brass disc of diameter $3.1 \mathrm{~cm}$ with a hole of diameter 1.4 $\mathrm{cm}$ cut in the center) was put on top of the skin sample and the ointment was applied to the surface of the stratum corneum and troweled flush with the edge of the template with the aid of the straight edge of a square cover glass. The template was then carefully removed and the skin membrane was mounted on a Franz diffusion cell (Crown glass, Somerville, NJ) with the formulation exposed. The receiver compartment was filled with $\mathrm{pH} 7$ phosphate buffer and maintained at $37^{\circ} \mathrm{C}$ with a jacket of circulating water. The receiver was continuously stirred with a bar magnet.

For the permeation of drugs from solution, an appropriately sized section of skin was cut and directly mounted in a Franz diffusion cell with the stratum corneum facing upwards. After the receiver compartment was filled with phosphate buffer to the predetermined operational level, 80 $\mu 1$ of the drug containing vehicle were applied to the skin surface with a micropipet.

The moment a skin membrane with the ointment was properly positioned and the moment of applying an aliquot of the solution formulation marked the "zero time" for each run. At predetermined time intervals, $0.6 \mathrm{ml}$ samples were withdrawn from the receiver compartment by a 1-ml tuberculin syringe fitted with a 23-gauge needle in turn fitted with a section of PE-50 polyethylene tubing. Each sample removed was replaced with an equal volume of phosphate buffer. Exactly $0.5 \mathrm{ml}$ of each sample were pipetted into $10 \mathrm{ml}$ liquid scintillation fluid (Safety Solve, RPI, Mount Prospect, IL) and assayed on a scintillation counter.

\section{Analysis of data}

After correction for the dilution of sampling, the data were plotted as the cumulative amount collected in the receiver compartment as a function of time. The flux into the receiving reservoir in the quasi-steady-state is equal to:

$$
J=\frac{V}{A} \cdot \frac{\mathrm{d} C}{\mathrm{~d} t}
$$

where $J$ is the total pseudo-steady-state flux $\left(\mathrm{mg} / \mathrm{cm}^{2} / \mathrm{h}\right)$ across the skin; $A$ is the area for diffusion $\left(\mathrm{cm}^{2}\right) ; V$ is the volume of the receiver compartment $(\mathrm{ml})$; and $\mathrm{d} C / \mathrm{d} t$ is the steady-state slope $(\mathrm{mg} / \mathrm{ml} / \mathrm{h})$. Slopes were calculated using least-squares linear regressions of the apparent steady-states in and around the $24 \mathrm{~h}$ point for the hydrocortisone formulations and for the minoxidil ointment. To gain working estimates of the fluxes from minoxidil solutions, the fluxes were estimated from the slopes of statistically drawn straight lines through all data points on the curvilinear profiles between 8 and 24 h. Generally, data past $24 \mathrm{~h}$ were only included in the calculation of fluxes if they fell on steady-state portions of the profiles begun within the first $24 \mathrm{~h}$. This placed a very conservative bias on the estimated fluxes from minoxidil solutions.

\section{Results}

Representative permeation profiles of minoxidil and hydrocortisone from the ointment and solution vehicles are shown in Figs. 1 and 2, respectively. It can be seen that after an initial lag period, the drugs permeated the skin from both vehicles in easily measured amounts. The permeation rates of both drugs attained steady levels

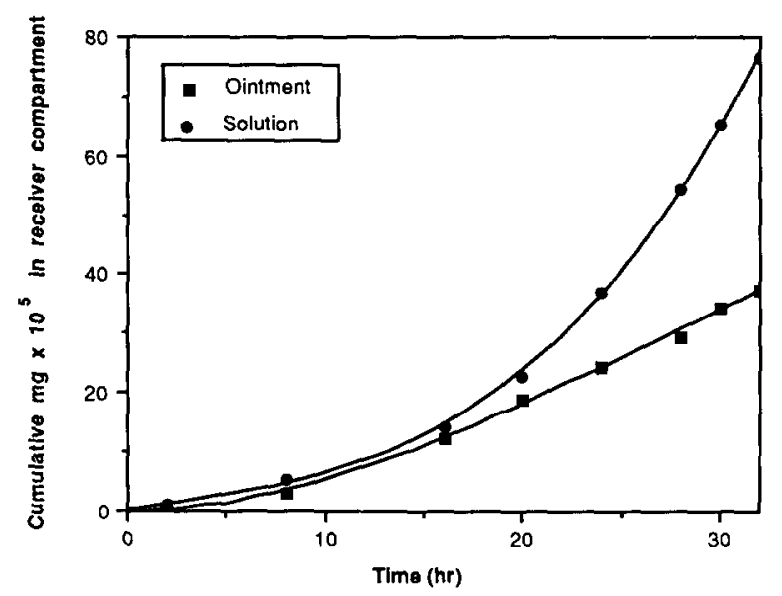

Fig. 1. Representative plots of permeation of $2 \%$ minoxidil from ointment and solution vehicles through human cadaver skin. 


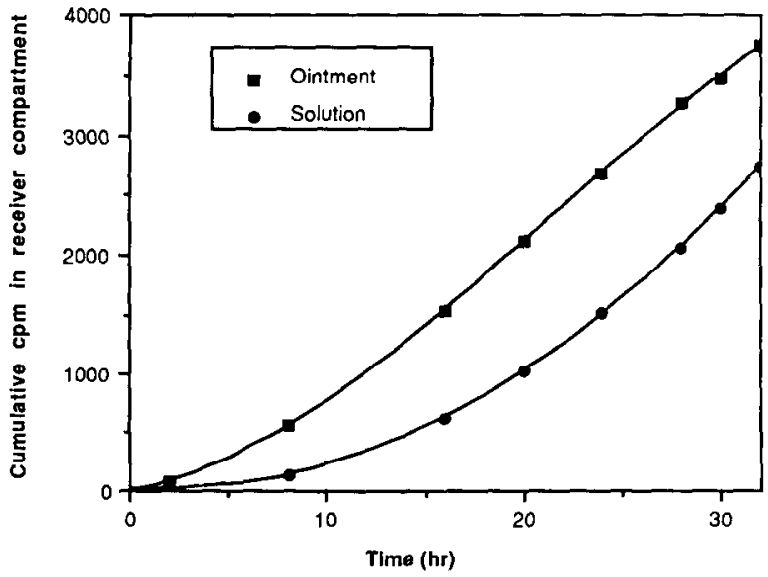

Fig. 2. Representative plots of permeation of tracer hydrocortisone from ointment and solution vehicles through human cadaver skin.

from the ointment. The solution vehicle, however, gave curvilinear profiles with particularly striking curvature in the case of minoxidil. For hydrocortisone formulations and minoxidil in the ointment,

\section{TABLE 1}

Fluxes of minoxidil (2\%) and hydrocortisone (trace amounts) from solution and ointment vehicles through human cadaver skin determined by finite dose experiments

\begin{tabular}{|c|c|c|c|}
\hline $\begin{array}{l}\text { Cadaver } \\
\text { no. }\end{array}$ & Ointment & Solution & $\begin{array}{l}\text { Solution/ } \\
\text { Ointment }\end{array}$ \\
\hline \multicolumn{4}{|c|}{ Flux of minoxidil $\left(\times 10^{5} \mathrm{mg} / \mathrm{cm}^{2} / \mathrm{h}\right)$} \\
\hline 1 & $0.51^{\mathrm{a}}$ & $1.3 \pm 0.43$ & 2.5 \\
\hline 2 & $0.37(0.38,0.37)^{b}$ & $1.3 \pm 0.1$ & 3.5 \\
\hline 3 & $2.2 \pm 0.97^{\mathrm{c}}$ & $7.3 \pm 3.3$ & 3.4 \\
\hline 4 & $7.8 \pm 2.67$ & $10.0 \pm 2.8$ & 1.3 \\
\hline 5 & $1.6(2.5,0.70)$ & $4.4(4.9,3.8)$ & 2.8 \\
\hline 6 & $9.2 \pm 2.0$ & $18.7 \pm 2.0$ & 2.0 \\
\hline 7 & $3.8 \pm 0.38$ & $9.0 \pm 2.4$ & 2.4 \\
\hline 8 & $4.8 \pm 0.36$ & $13.6 \pm 3.2$ & 2.8 \\
\hline \multicolumn{3}{|c|}{ Mean \pm S.D. } & $2.6 \pm 0.7$ \\
\hline \multicolumn{4}{|c|}{ Flux of hydrocortisone $\left(\mathrm{cpm} / \mathrm{cm}^{2} / \mathrm{h}\right)$} \\
\hline 2 & $983 \pm 235$ & $946 \pm 83$ & 1.0 \\
\hline 3 & $696 \pm 269$ & $966 \pm 337$ & 1.4 \\
\hline 4 & $1335 \pm 2.70$ & $1291 \pm 477$ & 1.0 \\
\hline 6 & $991 \pm 243$ & $1166 \pm 72$ & 1.2 \\
\hline \multicolumn{3}{|c|}{ Mean \pm S.D. } & $1.1 \pm 0.2$ \\
\hline
\end{tabular}

a Single experiment.

${ }^{b}$ Mean with individual values in parentheses; 2 experiments.

c Mean \pm S.D.; 3 or more experiments. the best straight line possible was drawn through the data to the end of the experiments at $32 \mathrm{~h}$. Since a steady-state region was not apparent for the minoxidil solution permeation curves, the 8-24 $h$ region was used to generate functional flux values. Earlier time points were passed over to recognize the lag time and later time points were discarded as being impractically long.

The fluxes for minoxidil and hydrocortisone from the two formulations calculated as described above and the calculated ratio of the fluxes for each drug from these two formulations are listed in Table 1.

\section{Discussion}

As previously demonstrated (Chiang et al. 1988), in vitro drug delivery discrimination is possible using 3 semi-solid topical formulations applied to a section of skin obtained from a single cadaver. However, there is a limit to the amount of information which can be gathered on a single sample of skin and one may question the representativeness of results and rankings established with skin from a single source. Since the work reported by Shaw et al. (1980) and Southwell et al. (1984) suggest that flux values obtained on skin samples from different donors likely do not provide valid comparisons and given the sparseness of credible data, in vitro methods to evaluate topical drug delivery can be called into question despite the fact that, in principle, the methods should be discerning. These issues are addressed in the present study.

The flux of minoxidil from the ointment (Fig. 1) attains a steady-state after an initial lag period of between 6 and $8 \mathrm{~h}$. However, steady delivery (throughput) is not apparent for the minoxidil solution. The steady-state for the ointment was not unexpected since minoxidil was present in amounts greatly exceeding its solubility in the vehicle and since the vehicle is compositionally stable during the course of the experiment. On the other hand, for the minoxidil solution formulation, the concentration of drug and composition of solvents vary systematically upon application to the skin. Hence, the thermodynamic activity of the 
drug would be expected to radically change over the course of an experiment. Specifically, the vehicle composition varies as the alcohol and water evaporate in accordance with their respective vapor pressures. In fact, when the alcohol evaporates (within a few hours), the resultant solution is at or possibly slightly above its saturation value (precipitation of drug can be brought about by seeding a 3-5\% solution under identical conditions). Comparable continuous curvature is not seen for the hydrocortisone solution apparently because the thermodynamic activity remains about the same even during the vehicle's evaporation since extremely dilute solutions were used (trace amounts).

For the 8 human cadaver abdominal skin sections studied, the flux of minoxidil from the ointment varied from $0.37 \times 10^{-5} \mathrm{mg} / \mathrm{cm}^{2} / \mathrm{h}$ to $9.2 \times$ $10^{-5} \mathrm{mg} / \mathrm{cm}^{2} / \mathrm{h}$, a 25 -fold spread (Table 1). The fluxes of minoxidil from the solution vehicle varied from $1.25 \times 10^{-5} \mathrm{mg} / \mathrm{cm}^{2} / \mathrm{h}$ to $18.7 \times 10^{-5}$ $\mathrm{mg} / \mathrm{cm}^{2} / \mathrm{h}$, a 15 -fold spread. The minoxidil flux data were evaluated statistically utilizing two-factor analysis of variance (ANOVA). Differences between formulations and among cadavers were significant $(P<0.0001$ for each effect). These differences, however, could not be legitimately evaluated due to the presence of a significant interaction between the two factors $(P<0.034)$. The presence of interaction was not unexpected as the minoxidil ointment and solution flux ratios (rather than flux differences) tended to be constant across cadavers. Ratios of the average formulation flux values, when calculated across cadavers (see Table 1), varied from 1.29 to 3.52 (only a 3-fold spread). A logarithmic transformation of the data yielded a two factor ANOVA model in which the cadaver/formulation interaction term was not significant $(P>0.3)$. Hence, the cadaver and formulation main effects could be appropriately evaluated. Differences among cadavers and between formulations were significant $(P<0.001$ for each effect.).

The flux of hydrocortisone from the ointment varied from $696 \mathrm{cpm} / \mathrm{cm}^{2} / \mathrm{h}$ to $1355 \mathrm{cpm} / \mathrm{cm}^{2} / \mathrm{h}$, a two-fold spread, using the 4 human cadaver abdominal skin samples studied. The fluxes of hydrocortisone from the solution vehicle varied from $946 \mathrm{cpm} / \mathrm{cm}^{2} / \mathrm{h}$ to $1291 \mathrm{cpm} / \mathrm{cm}^{2} / \mathrm{h}$, a $1.4-$ fold spread. Statistical analysis of the hydrocortisone flux data by two-factor ANOVA indicated that differences between formulations were not significant $(P>0.4)$, but that differences among cadavers were significant $(P<0.03)$. The ratios of average hydrocortisone flux values, when calculated across cadavers (see Table 1), varied from 0.96 to 1.39 , suggesting little difference between formulations.

These results suggest that a large degree of inter-cadaver variation in in vitro flux can exist for certain compounds. In light of this, the evaluation of formulation differences may be impossible for these compounds, unless comparisons are made within sets of skin sections taken from the same cadaver. Using our previously described experimental technique, formulations which differed in in vitro flux by a factor as small as 2 or 3 were successfully evaluated, and hence it is possible to grade the performance of topical formulations. This can be best achieved by including a reference standard formulation, evaluated in a matched piece of skin from the same cadaver, in each evaluation of a test formulation. The average flux value of the test formulation can then be expressed relative to the average flux value for the reference formulation. This ratio appears to be independent of the cadaver skin sample utilized, and hence can be used to compare a given test formulation against the relative flux values of other test formulations studied in a similar manner.

Finally, it should be pointed out that a considerable degree of arbitrariness is associated with the evaluation of data as these. For instance, the above comparisons are based on effective fluxes of the permeants achieved in the $8-24 \mathrm{~h}$ period of the experiments. Had the cumulative amount delivered in a fixed time, i.e. for $12 \mathrm{~h}$, been chosen as the indicator of delivery, one might conclude that the minoxidil formulations but not the hydrocortisone formulations were comparable in delivery (see Figs. 1, 2). Putting the delivery in terms of the amount accumulated in any fixed point in time suffers from the problem that variation in lag time distorts the delivery comparison even more than the failure to achieve a steady state flux tends to distort it. Thus, as long as lag times are on the 
short side of a period of application which is clinically realistic, here judged to be $24 \mathrm{~h}$, fluxes seem to offer the more telling indication of relative delivery merit. Comparisons of fluxes or any other measures of delivery based on times far exceeding $24 \mathrm{~h}$ raise serious questions because such periods seem unrealistically long relative to the normal patterns of use of ointment and creams. It should be noted that in the present case with curvilinear data, had any other reasonable method been used to estimate fluxes, i.e. the instantaneous slope at $24 \mathrm{~h}$ or use of late developing (post $24 \mathrm{hr}$ ) steady-states, differences in delivery would have been unduly exaggerated.

\section{Acknowledgements}

This study was supported by The Upjohn Co., Kalamazoo, MI, and by Grant AI/GM22303 from the National Institute of Health.

\section{References}

Chiang, C.-M., Flynn, G.L. Weiner, N.D., Addicks, W.J. and Szpunar, G.J., Int. J. Pharm., (1988) in press.

Shaw, J.E., Taskowich, L. and Chandrasekaran, S.K., In Current Concepts in Cutaneous Toxicity, Academic, New York, (1980) pp. 127-133.

Southwell, D., Barry, B.W. and Woodford, R., Int. J. Pharm., 3 (1984) 299-309. 